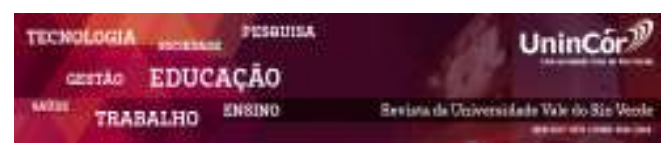

Revista da Universidade Vale do Rio Verde ISSN: 1517-0276 / EISSN: 2236-5362 Vol. 16 | n. 2 | Ano 2018

Babieli Corsini Baccoli Universidade do Vale do Sapucaí (UNIVÁS) babibaccoli@hotmail.com

Dênia Amélia Novato Castelli Von Atzingen Universidade do Vale do Sapucaí (UNIVÁS) denianovato@gmail.com

Adriana Rodrigues dos Anjos Mendonça Universidade do Vale do Sapucaí (UNIVÁS) drijar@hotmail.com

\section{PRÁTICA ESTÉTICA E A AUTOESTIMA DE PACIENTES EM TRATAMENTO ONCOLÓGICO}

\begin{abstract}
RESUMO
O câncer constitui um problema de saúde pública no Brasil, bem como mundialmente, agravado nos últimos anos devido ao envelhecimento populacional, principalmente em países em desenvolvimento. Essa é uma doença diferenciada de outras enfermidades crônicas em virtude de sua patologia que pode provocar deformidades, dor e mutilações, provocando, também, grande impacto psicológico, gerando a sentimentos negativos desde o momento do diagnóstico. A autoestima corresponde à valorização intrínseca que o indivíduo faz de si mesmo em diferentes situações e eventos da vida a partir de um determinado conjunto de valores eleitos por ele como positivos ou negativos. Assim, o objetivo do estudo foi avaliar se os procedimentos estéticos podem contribuir para a manutenção e/ ou elevação da autoestima de pacientes em tratamento oncológico. Participaram dessa pesquisa 32 mulheres em tratamentos de quimioterapia e radioterapia. O presente estudo foi de abordagem quantitativa, descritiva, longitudinal, com amostragem aleatória simples e foi desenvolvido na Associação do Voluntariado de Varginha "Vida Viva". Os resultados obtidos mostraram que os pacientes apresentaram autoestima inicial de 21,84 e final de 21,47. Não foram encontradas diferenças entre a autoestima antes e após a aplicação dos procedimentos estéticos. Conclui-se que as práticas estéticas contribuíram para a manutenção da autoestima dos pacientes.
\end{abstract}

Palavras-chave: Bioética. Autoestima. Estética. Câncer.

\section{PRACTICE AND THE SELF-ESTEEM OF PATIENTS IN ONCOLOGICAL TREATMENT}

\begin{abstract}
Cancer is a public health problem in Brazil, as well as worldwide, getting worsened in recent years due to the population aging, especially in developing countries. Cancer is distinguished of other chronical diseases as its pathology can cause deformities, pain and mutilations, tauting, in addition, great psychological impact, generating to negative feelings from the moment of diagnosis. Self-esteem corresponds to the intrinsicappreciation that individuals make of themselves in different situations and events of life from a certain set of values chosen by them as positive or negative. Thus, the objective of this study was to evaluate if the aesthetic procedures can contribute to the maintenance and/ or elevation of the self-esteem of patients undergoing oncological treatment. Thirty-two women who were undergoing chemotherapy and radiotherapy treatmentstook part in that study. The present study was a quantitative, descriptive, longitudinal, approach with simple random sampling and it was developed at the Volunteer Association "Vida Viva", in Varginha.
\end{abstract}


The observed results have shown that patients presented initial self-esteem of 21.84 and finalself-esteem of 21.47. No differences were obtained between self-esteem before and after the application of aesthetic procedures. It has been concluded that the aesthetic practices contributed to the maintenance of patients' selfesteem.

Keywords: Bioethics. Self-esteem. Aesthetics. Cancer.

\section{Recebido em: 22/06/2018 - Aprovado em: 30/08/2018 - Disponibilizado em: 15/12/2018}

\section{INTRODUÇÂO}

A beleza é algo que chama a atenção do homem desde o início dos tempos, seja na literatura, na pintura, na arte ou no próprio homem. A beleza humana sempre foi cultuada, conforme as imposições de sua época (VIGARELLO, 2006).

A estética corresponde ao estudo da beleza e do belo; seria tudo o que tem propriedade de beleza, sendo muitas vezes percebida durante a análise da aparência física, pois o belo se define como algo "que tem forma ou aparência agradável, perfeita, harmoniosa, que desperta sentimentos de admiração, de grandeza, de nobreza, de prazer, de perfeição" (VILAS BOAS, 2015).

Já é sabido que a vaidade é uma marcante característica do povo brasileiro, e o campo da estética é bastante amplo, por este motivo justificam-se os procedimentos e tratamentos de embelezamento que mais influenciam na automotivação e autoestima dos seres humanos (CURY, 2005).

Enfrentar o câncer já exige força interior muito grande, mas, além da doença, grande parte dos pacientes sofre com os efeitos colaterais da quimioterapia e da radioterapia que modificam a aparência, como a queda dos cabelos e pelos do corpo, ressecamento da pele e perda ou ganho de peso. Especialistas reconhecem que manter a autoestima é fundamental para aumentar a tolerância ao tratamento, com influência até mesmo no resultado terapêutico (DAHER, 2013).

Os profissionais da estética podem orientar a reconstrução e valorização da autoimagem e confiança, possibilitando que o paciente se concentre primeiramente na recuperação da sua doença e na retomada de sua saúde, e também obtenha informações sobre cuidados da estética, opções que se dispõem a cuidar da pele que sofre alterações como queimadura e ressecamento e das unhas que ficam enfraquecidas durante a quimioterapia. Esses pequenos cuidados podem ajudar as pessoas que estão em tratamento contra o câncer a sentirem-se melhor (PIETRUK et al., 2009).

Com isso, o objetivo do presente estudo visa avaliar se os procedimentos estéticos podem contribuir para a manutenção e/ou elevação da autoestima de pacientes em tratamento oncológico.

Intimamente ligados ao conceito de beleza, existem vários centros ou clínicas de estética, onde pessoas podem fazer vários tratamentos com o objetivo de melhorar a sua aparência física (MELO, 2016). 
Os cosméticos ganham cada vez mais importância na dermatologia, firmando mundialmente o Brasil como terceiro maior mercado consumidor (NASCIMENTO, 2003).

A popularização da medicina da beleza e os excessos por ela estimulados têm motivado não só os pacientes, mas também alguns médicos a pensar no corpo como uma obra inacabada que deve ser constantemente melhorada (SCHMIDT, 2015).

Para Branden (1995), ainda partindo da ideia de a autoestima ser uma poderosa necessidade humana que contribui de maneira essencial para o processo da vida, ela é indispensável para um desenvolvimento normal e saudável. A autoestima mostra um conceito positivo de si próprio, é ter a capacidade de enxergar a beleza e as qualidades do indivíduo.

O profissional de estética é responsável por cuidar da saúde do corpo e da pele, voltandose para o bem-estar físico, estético e mental das pessoas. Caracterizado por lidar com mulheres ou homens, esse profissional pode se especializar e atuar em diversas áreas (FONSECA, 2017).

Sabendo-se que o paciente pode ter seu equilíbrio psicológico ameaçado pelas mudanças que certamente ocorrem no período da doença e dos tratamentos, incluindo alterações em sua autoestima, a adaptação ou o ajuste psicossocial ao câncer é um processo durante o qual cada pessoa procura controlar seus sofrimentos, resolver problemas específicos e alcançar algum controle sobre acontecimentos desencadeados pela doença (SOUZA; ARAUJO, 2010).

Durante a quimioterapia e a radioterapia a pele fica mais ressecada e sensível, por isso qualquer procedimento estético que o paciente quiser fazer tem que ter recomendação e acompanhamento médico. Alguns são, inclusive, proibidos. Procedimentos que necessitam uso de agulhas, por exemplo, não podem ser feitos porque aumentam o risco de infecção. Limpeza de pele, peelings, tinturas de cabelo também devem ser evitados (NIARA, 2017).

O paciente com câncer poderá ser tratado e se submeter a qualquer tipo de tratamento, pelo menos em doses terapêuticas e por um tempo considerado normal de acompanhamento, fazendo uso de procedimentos estéticos. Para quem tem a doença, seguem as técnicas estéticas que podem ser utilizadas: designer de sobrancelha e sobrancelha de hena - técnica utilizada com intuito de corrigir pequenas falhas das sobrancelhas -, higienização facial - realizado com leite de limpeza e loção tônica como parte dos cuidados diários com a pele -, hidratação cutânea realizada com creme neutro para auxiliar o papel da pele nas suas funções mantendo íntegro o sistema de defesa do organismo -, aplicação de protetor solar para a prevenção do câncer de pele. Além das práticas estéticas, são feitas demonstrações de uso de lenços na cabeça, incentivando as pacientes, com o fito de amenizar a dor e as marcas deixadas pelo tratamento contra o câncer, tendo a autoestima em alta e sentindose belas (INCA, 2017).

De acordo com o INCA (Instituto Nacional de Câncer), "O câncer é o nome dado a um conjunto de mais de 100 doenças que têm em comum o crescimento desordenado (maligno) de células que invadem os tecidos e órgãos, podendo espalhar-se (metástase) para outras regiões do corpo" (INCA 2017). 
Segundo Santana, et al. (2008), o câncer é uma doença cujo tratamento é doloroso e seus efeitos colaterais desencadeiam mudanças na vida do indivíduo. Em muitos casos, ele perde sua independência, sofre com alterações em sua imagem corporal, se isola de seus vínculos sociais, se afasta de atividades de lazer e apresenta sentimento de inutilidade.

O abalo psicológico causado pelo câncer traz uma significativa repercussão na vida da paciente. Quando esse momento é vivido com conhecimento e compreensão, contando com um apoio psíquico, torna-se possível o entendimento dos seus medos e angústias que podem interferir em uma resposta ao seu tratamento terapêutico (AMARANTE, 2015).

Ainda a autoestima poderá mostrar o sentimento, apreço e importância que a pessoa sente por si própria, sendo o centro de sua vida subjetiva, e destinando seu pensamento e comportamento (ISHIZUKA, 2012).

\section{MÉTODOS}

Tipo de estudo: $O$ presente estudo foi de abordagem quantitativa, descritiva, longitudinal, com amostragem aleatória simples.

Local do Estudo: O estudo foi desenvolvido na Associação do Voluntariado de Varginha "Vida Viva", associação civil, sem fins lucrativos, fundada em 22/07/1996 por Lígia Inês Braga e Meryvone Mansur Bíscaro, e que presta atendimento a aproximadamente 2.600 pacientes oncológicos da cidade de Varginha e mais 198 municípios que fazem tratamento no hospital Bom Pastor. Mantém horário de atendimento das
$8 \mathrm{~h}$ às $18 \mathrm{~h}$ e sua missão é promover gratuitamente o atendimento assistencial aos pacientes oncológicos de todas as faixas etárias da cidade de Varginha e região. Seu atendimento socioassistencial reúne profissionais de Serviço Social, Psicologia, Farmácia e Direitos Jurídicos e oferece também um espaço adequado às atividades profissionais dos Fisioterapeutas e Esteticistas. É uma das entidades de Minas Gerais escolhidas pela ONU para participar do programa de Voluntários das Nações Unidas. Esses pacientes procuram a instituição com o intuito de acolhimento, pois a unidade deixa à disposição dos usuários tvs com poltronas reclináveis para descanso, banheiros e salas de entretenimento, proporcionando, dessa forma, um espaço agradável e acolhedor aos pacientes em tratamento na cidade e aos seus acompanhantes.

Participantes da Pesquisa: 32 mulheres em tratamentos de quimioterapia e radioterapia.

Critérios de Inclusão: Mulheres entre 18 a 60 anos de idade, que apresentaram diagnóstico de câncer, com recomendação de tratamento quimioterápico ou radioterápico e que assinaram o TCLE. Essas pacientes oncológicas estão em tratamento quimioterápico ou radioterápico em decorrência de variados tipos de câncer, como: mama; intestino; ossos; pulmão; útero; estômago e esôfago. A idade foi delimitada devido ao público alvo dessa faixa etária.

Critérios de Não Inclusão: Participantes com diagnóstico de câncer, mas que não estivessem em tratamentos quimioterápico ou radioterápico e as que apresentassem qualquer tipo de reação alérgica já conhecida aos produtos utilizados nas técnicas de estética. 
Critérios de Exclusão: Participantes que solicitassem deixar a pesquisa a qualquer momento, que faltassem ao tratamento estético e que apresentassem qualquer tipo de reação alérgica aos produtos utilizados nas técnicas de estética. Não houve exclusão de pacientes.

Instrumentos de Pesquisa: Instrumento de dados sociodemográficos e Escala de Autoestima de Rosenberg - EPM.

Procedimentos para Coleta de Dados: As participantes da pesquisa que estavam em tratamento oncológico no "Vida Viva" em Varginha foram convidadas a participarem da pesquisa após serem informadas de seus objetivos e conteúdo. Após a concordância, foi solicitado que elas assinassem o Termo de Consentimento Livre e Esclarecido. A pesquisadora procurou um local tranquilo e sem interferência de outras pessoas e iniciou o trabalho com o preenchimento do Instrumento de Dados Sócio Demográficos e, em seguida, as perguntas que integram a Escala de Avaliação de Autoestima de Rosenberg - EPM. Após esse procedimento, deu-se início a aplicação das técnicas de estética, que foram realizadas uma vez por semana com cada participante, durante dois meses, perfazendo um total de 08 sessões por participante. Esse número de sessões foi escolhido como sendo ideal para que houvesse uma melhora geral no aspecto da pele das participantes. As técnicas estéticas utilizadas foram:

1) Designer de sobrancelha e sobrancelha de hena: técnica utilizada com intuito de corrigir pequenas falhas das sobrancelhas;
2) Higienização facial: realizada com leite de limpeza e loção tônica como parte dos cuidados diários com a pele;

3) Hidratação cutânea: realizada com creme neutro para auxiliar o papel da pele nas suas funções, mantendo íntegro o sistema de defesa do organismo;

4) Aplicação de protetor solar para a prevenção do câncer de pele.

Ao final das 08 sessões, foi aplicada novamente a Escala de Autoestima de Rosenberg.

Análise dos Dados: Foram realizadas as análises estatísticas descritivas e inferenciais. $\mathrm{Na}$ análise descritiva, as variáveis quantitativas (idade, número de filhos e Escala de Autoestima de Rosenberg.) foram apresentadas através de medidas de tendência central (média e mediana) e por medidas de dispersão (desvio padrão). A distribuição dessas variáveis foi avaliada por testes de aderência a normalidade. As variáveis categóricas (faixas de idade, estado civil, com quem reside, se tem filhos, nível de escolaridade e tempo de diagnóstico da doença) foram descritas através de tabelas de frequências (proporções). Na etapa da análise inferencial foi utilizado o Teste Não Paramétrico de Wilcoxon, para avaliar a diferença entre a avaliação inicial e a avaliação final da Escala de Autoestima de Rosenberg. As diferenças entre as categorias de respostas das variáveis categóricas em relação à Escala de Autoestima de Rosenberg, foram avaliadas através do Teste Não Paramétrico de Kruskal-Wallis.

Procedimentos Éticos: Essa pesquisa seguiu as determinações da Resolução $\mathrm{N}^{\circ}$ 466, de 12 de dezembro de 2012, do Conselho Nacional de 
Saúde (CNS) que define os procedimentos éticos para a pesquisa em seres humanos. $O$ trabalho somente foi iniciado após aprovação do Comitê de Ética e Pesquisa, conforme Parecer Consubstanciado $\mathrm{n}^{\mathrm{o}} 2.015 .400$.

\section{RESULTADOS E DISCUSSÃO}

Aqui serão apresentados os resultados dos dados Sociodemográficos e logo a seguir os resultados da Escala de Autoestima de Rosenberg.

O estudo analisou e concluiu que não há correlação estatisticamente significante entre a Idade das pacientes e as Escalas de Autoestima de Rosenberg Inicial $(\mathrm{p}=0,744)$ e Final $(\mathrm{p}=$ $0,871)$.
$\mathrm{Na}$ tabela 1, observou que entre os participantes a faixa de idade é de 47 a 55 anos $28,1 \%$. Já a faixa do estado civil prevaleceu de 15 com 46,9\%. Com quem reside a maioria foi de 15 com 46,9\%. Ter filhos a maioria respondeu que "Sim" 27 com 84,4\%, A escolaridade a maioria tem $1^{\circ}$ grau incompleto 19 com $59,4 \%$. No tempo de diagnóstico da doença, a maioria é para "outras idades" 16 com 50\%.

Tabela 1 - Demonstrativos sociodemográficos dos participantes

\begin{tabular}{|c|c|c|}
\hline Variáveis & $\mathbf{N}$ & $\%$ \\
\hline \multicolumn{3}{|l|}{ Faixas de Idade } \\
\hline 26 a 46 anos & 8 & 25,0 \\
\hline 47 a 55 anos & 9 & 28,1 \\
\hline 56 a 58 anos & 8 & 25,0 \\
\hline 59 a 60 anos & 7 & 21,9 \\
\hline \multicolumn{3}{|l|}{ Estado Civil } \\
\hline Solteira & 5 & 15,6 \\
\hline Casada & 15 & 46,9 \\
\hline Separada & 3 & 9,4 \\
\hline Viúva & 9 & 28,1 \\
\hline \multicolumn{3}{|l|}{ Com quem reside } \\
\hline Esposo & 15 & 46,9 \\
\hline Filhos & 10 & 31,3 \\
\hline Pais & 1 & 3,1 \\
\hline Outros & 6 & 18,8 \\
\hline \multicolumn{3}{|l|}{ Filhos } \\
\hline Sim & 27 & 84,4 \\
\hline Não & 5 & 15,6 \\
\hline \multicolumn{3}{|l|}{ Escolaridade } \\
\hline $1^{\circ}$ Grau completo & 4 & 12,5 \\
\hline $1^{\circ}$ Grau incompleto & 19 & 59,4 \\
\hline $2^{\circ}$ Grau completo & 5 & 15,6 \\
\hline
\end{tabular}




\begin{tabular}{lcc}
$2^{\circ}$ Grau incompleto & 1 & 3,1 \\
Graduação completa & 2 & 6,3 \\
Graduação incompleta & 1 & 3,1 \\
\hline Tempo de diagnóstico da doença & & 3,1 \\
2 a 4 meses & 1 & 9,4 \\
4 a 6 meses & 3 & 21,9 \\
a 8 meses & 7 & 9,4 \\
8 a 10 meses & 3 & 6,3 \\
10 a 12 meses & 2 & 50,0 \\
Outros & 16 & $\mathbf{1 0 0 , 0}$ \\
Total & $\mathbf{3 2}$ & $\mathbf{1 6}$ \\
\hline \hline
\end{tabular}

Fonte: o próprio autor

Na tabela 2, com relação à idade, os 51,63 anos e mediana de 55. Números de filhos participantes da pesquisa apresentaram média de com a média de 2,19 e mediana de 2 .

Tabela 1 - Média, Mediana e desvio padrão em relação às variáveis idade e número de filhos

\begin{tabular}{lcccc}
\hline \multicolumn{1}{c}{ Variáveis } & $\mathbf{N}$ & Média & Mediana & $\begin{array}{c}\text { Desvio- } \\
\text { Padrão }\end{array}$ \\
\hline Idade & 32 & 51,63 & 55 & 9,136 \\
Número de filhos & 32 & 2,19 & 2 & 1,749 \\
\hline \hline
\end{tabular}

Fonte: o próprio autor

A Tabela 3 traz os resultados obtidos na Escala de Rosenberg antes e depois do procedimento estético. Fazendo a média dos resultados, pode-se notar que há uma diferença muito pequena assim como a mediana e o desvio padrão. Em ambos os casos, em média, os participantes apresentam uma autoestima equilibrada (entre 15 e 25 ).

Tabela 2 - Média, mediana e desvio de padrão em relação às variáveis Total inicial e Total final

\begin{tabular}{lccccc}
\hline \hline \multicolumn{1}{c}{ Escala de Rosenberg } & N & Média & Mediana & $\begin{array}{c}\text { Desvio- } \\
\text { Padrão }\end{array}$ & Valor-p* \\
\hline Total Inicial & 32 & 21,84 & 23 & 5,341 & 0,101 \\
Total Final & 32 & 21,47 & 21 & 4,711 & \\
\hline \hline
\end{tabular}

* Teste de Wilcoxon. Diferença estatisticamente não significante.

De acordo com os resultados encontrados, não houve correlação entre a idade das pacientes e autoestima $(\mathrm{p}=0,871)$.

Também não foram encontradas correlações entre as categorias de Estado Civil, em relação à avaliação inicial $(\mathrm{p}=0,884)$ e a avaliação final $(\mathrm{p}=0,970)$ da Escala de Autoestima de Rosenberg.
Foi encontrada uma correlação negativa entre o número de filhos e a Escala de Autoestima de Rosenberg, Inicial $(\mathrm{p}=0,010)$ e Final $(\mathrm{p}=0,037)$, isto é, quanto maior o número de filhos, menor tende a ser o valor da Escala de Autoestima de Rosenberg.

A tabela 04 mostra os resultados iniciais e finais da Escala de Autoestima de Rosenberg, 
ou seja, os resultados em número (n) e em

os procedimentos estéticos.

porcentagem $(\%)$ de cada questão, antes e após

Tabela 3 - Resultados da Escala de Autoestima de Rosenberg antes (avaliação inicial) e após (avaliação final) o procedimento Estético

\begin{tabular}{|c|c|c|c|c|c|c|c|c|c|c|}
\hline \multirow[t]{2}{*}{ Avaliação Inicial } & \multicolumn{2}{|c|}{$\begin{array}{c}\text { Discordo } \\
\text { Totalmente }\end{array}$} & \multicolumn{2}{|c|}{ Discordo } & \multicolumn{2}{|c|}{ Concordo } & \multicolumn{2}{|c|}{$\begin{array}{l}\text { Concordo } \\
\text { Totalmente }\end{array}$} & \multicolumn{2}{|c|}{ Total } \\
\hline & $\mathbf{n}$ & $\%$ & $\mathbf{n}$ & $\%$ & $\mathbf{N}$ & $\%$ & $\mathbf{n}$ & $\%$ & $\mathbf{n}$ & $\%$ \\
\hline $\begin{array}{l}\text { 1. Eu sinto que sou uma pessoa de } \\
\text { valor, no mínimo, tanto quanto as } \\
\text { outras pessoas. }\end{array}$ & 0 & 0,0 & 1 & 3,1 & 21 & 65,6 & 10 & 31,3 & 32 & 100,0 \\
\hline $\begin{array}{l}\text { 2. Eu acho que eu tenho várias boas } \\
\text { qualidades. }\end{array}$ & 0 & 0,0 & 3 & 9,4 & 18 & 56,3 & 11 & 34,4 & 32 & 100,0 \\
\hline $\begin{array}{l}\text { 3. Levando tudo em conta, eu penso } \\
\text { que eu sou um fracasso. }\end{array}$ & 14 & 43,8 & 6 & 18,8 & 10 & 31,3 & 2 & 6,3 & 32 & 100,0 \\
\hline $\begin{array}{l}\text { 4. Eu acho que sou capaz de fazer as } \\
\text { coisas tão bem quanto a maioria das } \\
\text { pessoas. }\end{array}$ & 0 & 0,0 & 2 & 6,3 & 15 & 46,9 & 15 & 46,9 & 32 & 100,0 \\
\hline $\begin{array}{l}\text { 5. Eu acho que eu não tenho muito } \\
\text { que me orgulhar. }\end{array}$ & 13 & 40,6 & 11 & 34,4 & 3 & 9,4 & 5 & 15,6 & 32 & 100,0 \\
\hline $\begin{array}{l}\text { 6. Eu tenho uma atitude positiva com } \\
\text { relação a mim mesmo. }\end{array}$ & 0 & 0,0 & 1 & 3,1 & 16 & 50,0 & 15 & 46,9 & 32 & 100,0 \\
\hline $\begin{array}{l}\text { 7. No conjunto, eu estou satisfeito } \\
\text { comigo. }\end{array}$ & 2 & 6,3 & 1 & 3,1 & 15 & 46,9 & 14 & 43,8 & 32 & 100,0 \\
\hline $\begin{array}{l}\text { 8. Eu gostaria de poder ter mais } \\
\text { respeito por mim mesmo. }\end{array}$ & 8 & 25,0 & 11 & 34,4 & 10 & 31,3 & 3 & 9,4 & 32 & 100,0 \\
\hline 9. Âs vezes eu me sinto inútil. & 13 & 40,6 & 9 & 28,1 & 8 & 25,0 & 2 & 6,3 & 32 & 100,0 \\
\hline $\begin{array}{l}\text { 10. Às vezes eu acho que eu não } \\
\text { presto pra nada. }\end{array}$ & 19 & 59,4 & 8 & 25,0 & 3 & 9,4 & 2 & 6,3 & 32 & 100,0 \\
\hline
\end{tabular}

\begin{tabular}{|c|c|c|c|c|c|c|c|c|c|c|}
\hline \multirow[t]{2}{*}{ Avaliação Final } & \multicolumn{2}{|c|}{$\begin{array}{c}\text { Discordo } \\
\text { Totalmente }\end{array}$} & \multicolumn{2}{|c|}{ Discordo } & \multicolumn{2}{|c|}{ Concordo } & \multicolumn{2}{|c|}{$\begin{array}{c}\text { Concordo } \\
\text { Totalmente }\end{array}$} & \multicolumn{2}{|c|}{ Total } \\
\hline & $\mathbf{N}$ & $\%$ & $\mathbf{N}$ & $\%$ & $\mathbf{n}$ & $\%$ & $\mathbf{n}$ & $\%$ & $\mathbf{n}$ & $\%$ \\
\hline $\begin{array}{l}\text { 1. Eu sinto que sou uma pessoa de } \\
\text { valor, no mínimo, tanto quanto as } \\
\text { outras pessoas. }\end{array}$ & 1 & 3,1 & 1 & 3,1 & 21 & 65,6 & 9 & 28,1 & 32 & 100,0 \\
\hline $\begin{array}{l}\text { 2. Eu acho que eu tenho várias boas } \\
\text { qualidades. }\end{array}$ & 0 & 0,0 & 3 & 9,4 & 19 & 59,4 & 10 & 31,3 & 32 & 100,0 \\
\hline $\begin{array}{l}\text { 3. Levando tudo em conta, eu penso } \\
\text { que eu sou um fracasso. }\end{array}$ & 14 & 43,8 & 6 & 18,8 & 10 & 31,3 & 2 & 6,3 & 32 & 100,0 \\
\hline $\begin{array}{l}\text { 4. Eu acho que sou capaz de fazer as } \\
\text { coisas tão bem quanto as maioria das } \\
\text { pessoas. }\end{array}$ & 2 & 6,3 & 1 & 3,1 & 17 & 53,1 & 12 & 37,5 & 32 & 100,0 \\
\hline $\begin{array}{l}\text { 5.Eu acho que eu não tenho muito que } \\
\text { me orgulhar. }\end{array}$ & 11 & 34,4 & 12 & 37,5 & 5 & 15,6 & 4 & 12,5 & 32 & 100,0 \\
\hline $\begin{array}{l}\text { 6. Eu tenho uma atitude positiva com } \\
\text { relação a mim mesmo. }\end{array}$ & 0 & 0,0 & 1 & 3,1 & 18 & 56,3 & 13 & 40,6 & 32 & 100,0 \\
\hline $\begin{array}{l}\text { 7. No conjunto, eu estou satisfeito } \\
\text { comigo. }\end{array}$ & 1 & 3,1 & 2 & 6,3 & 17 & 53,1 & 12 & 37,5 & 32 & 100,0 \\
\hline $\begin{array}{l}\text { 8. Eu gostaria de poder ter mais } \\
\text { respeito por mim mesmo. }\end{array}$ & 7 & 21,9 & 12 & 37,5 & 11 & 34,4 & 2 & 6,3 & 32 & 100,0 \\
\hline 9. Ầs vezes eu me sinto inútil. & 10 & 31,3 & 15 & 46,9 & 6 & 18,8 & 1 & 3,1 & 32 & 100,0 \\
\hline $\begin{array}{l}\text { 10. Às vezes eu acho que eu não } \\
\text { presto pra nada. }\end{array}$ & 17 & 53,1 & 11 & 34,4 & 4 & 12,5 & 0 & 0,0 & 32 & 100,0 \\
\hline
\end{tabular}

Fonte: o próprio autor

Para saber como a estética afeta a autoestima das pessoas se faz necessário entender o que é automotivação e o que significa autoestima. Para Branden (2009, p.13) "a autoestima é a confiança em nossa capacidade para pensar e enfrentar os desafios da vida”. 
Em seu papel fundamental, a estética visa uma melhoria na qualidade de vida, oferecendo uma elevação da autoestima. Durante o tratamento contra o câncer, o comportamento da paciente influencia de forma significante nos seus resultados. A situação psicológica negativa colabora com o aumento da sensação de dor e maior desconforto físico (GOMES; SILVA, 2013; ALBUQUERQUE; PEREIRA, 2014).

Segundo Borba Thives, (2011) e

Pietruck et al. (2009), a autoestima em portadores de câncer poderia ser influenciada por profissionais da estética que orientariam a reconstrução e valorização da autoimagem e confiança, levando-os a concentrarem-se primeiramente na recuperação da sua doença e, na retomada da sua saúde, fornecendo-lhes informações e cuidados da estética, capazes de proporcionar-lhes maior conforto.

Ser paciente terminal evidencia a debilidade orgânica inerente à doença, que traz consigo, além dos aspectos físicos, todos os preconceitos de uma sociedade na qual a terminalidade afronta a negação da morte, mostrando que todos somos finitos (OLIVEIRA, 2002; BORGES et al., 2006).

Por meio dessa análise, o profissional de estética direciona o seu foco de ação a recursos terapêuticos e estéticos que visam amenizar possíveis intercorrências resultantes de cirurgias plásticas e estéticas e, desta forma, intensificar a busca pela qualidade de vida e ressaltar a autoestima dos pacientes (MEDEIROS, 1999; SANTANA, 2015).

Considerando essas transformações com as quais convivemos, somos favoráveis a uma bioética que se oriente pelo respeito e incentivo à liberdade individual de tomada de decisão, adicionada dos princípios da solidariedade, da justiça, da equidade e da responsabilidade, reforçando a necessidade de proteção dos mais desfavorecidos, vulneráveis, vulnerados ou frágeis. Uma bioética que auxilie a busca de soluções para conflitos entre liberdades individuais e interesses da coletividade, o respeito pelas liberdades e direitos individuais, os interesses da coletividade e a responsabilidade de proteger a saúde da coletividade.

\section{CONCLUSÃO}

Conclui-se que as práticas estéticas contribuíram para a manutenção da autoestima das pacientes.

\section{REFERÊNCIAS}

ALBUQUERQUE, Ana Claudia Alves; PEREIRA, Francielly Horst. Contribuindo para a melhoria da autoestima de mulheres com câncer de mama. Instituto Federal de Educação, Ciência e Tecnologia de Santa Catarina. Joinville: 2014.

AMARANTE, Suely. Aspectos psicológicos do câncer de mama. 2015. Disponível no site: http://portal.fiocruz.br/pt-br

BERTAN FC, CASTRO EK. Quality of Life, Anxiety and Depressions Indicators and Sexual Satisfaction in Adult Patients with Cancer. Rev Salud Soc; v.1, n.2, p.76-88. 2010.

BORBA, Tamila J. THIVES, Fabiana Marin. Uma reflexão sobre a influência da estética na autoestima, auto-motivação e bem estar do ser humano. Universidade do Vale do Itajaí UNIVALI, Santa Catarina: 2011 21p.

BORGES, Alini Daniéli Viana Sabino; SILVA, Elisângela Ferreira da; TONILLO, Patrícia 
Bighetti; MAZER, Sheila Maria Mazer; VALLE, Elizabeth Ranier Martins do; SANTOS, Manoel Antônio dos. Percepção da morte pelo paciente oncológico ao longo do desenvolvimento.

Psicologia em Estudo, Maringá, v. 11, n. 2, p. 361-369, mai./ago. 2006.

BRANDEN, Nathaniel. Auto-estima: como aprender a gostar de si. Editora Saraiva, São Paulo: 1995.

BRANDEN Nathaniel. O Poder da Autoestima. Editora Saraiva, São Paulo: 2009.

CURY, Augusto Jorge. A ditadura da beleza e a revolução das mulheres. Romance. Rio de Janeiro: Sextante, 2005.

DAHER, Daniela. Terapia da Beleza. Revista Rede Câncer. n.21, p. 03, abril, 2013.

FONSECA, Alessandra. O profissional de estética no mercado de trabalho. UNAMA. 2017.

GOMES N.S, SILVA S.R. Avaliação da autoestima de mulheres submetidas à cirurgia oncológica mamária. Texto Contexto Enferm; v.22, n.3, p.509-16. 2013.

INCA, Instituto nacional do câncer. Mantendo a Autoestima durante o tratamento Oncológico. Jundiaí, SP: 2017.

ISHIZUKA CK. Autoestima em pacientes submetidas a blefaroplastia. Rev Bras Cir Plást.; v.27, n.1, p.31-6. 2012.

MEDEIROS, Cyntia Galvão Gomes de. Princípios básicos de estética aplicados na dentística. Revista Robrac, v. 8, n. 25, p. 19-22, 1999.

MELO, Dannilo César Silva. Kovae Ta'angá Escolas Mbyá Guarani na Bienal do Mercosul: reflexões sobre educação e estética de colonial. 2016. 118 p.

NASCIMENTO, Leninha Valério. A beleza é supericial. Dermatológico. Fev. v. 78, n. 1, p. 119-120, 2003.

NIARA, Sonia. Tratamento estético durante a quimioterapia. 2017. Disponível no site:https://sonianiara.com/2017/01/17/tratament o-estetico-durante-a-quimioterapia
OLIVEIRA, Nucia Alexandra Silva de. Em jogo... os jogos da beleza. Revista de Estudos Femininos. v.10, n.1, p.254-6. jan. 2002.

PIETRUK, Cristiane Mary Kolbe; CASTELLANO, Mônica Fabiana; OLIVEIRA, Silvia Patrícia de. O Papel do Tecnólogo em Estética e Imagem Pessoal na Melhoria da Auto-Estima de Mulheres em Tratamento contra o Câncer de Mama. Curitiba, PR: 2009.

SANTANA, J. J. R. A.; ZANIM, C. R.; MANIGLIA, J. V. Pacientes com câncer: enfrentamento, rede social e apoio social. Paidéia, v.18, n. 40, p. 372-384, 2008.

SANTANA, A.L. Estética 2015. Disponível no site:

http://www.consciencia.net/filosofia/estetica.htm 1

SCHMIDT, Adriana. A medicina e a beleza. Curitiba: 2015. Disponível no site: http://www.adrianaschmidt.com/qualidade-devida-e-beleza/a-medicina-e-a-beleza/

SOUZA JR, ARAÚJO TCCF. Eficácia terapêutica de intervenção em grupo psicoeducacional: um estudo exploratório em oncologia. Estudo Psicol; v.27, n.2, p.187-96. 2010.

TEIXEIRA, Sérgio Alves. Produção e consumo social da beleza. Horizontes Antropológicos, v.7, n. 16, p. 189-220. dez 2001.

VIGARELLO, Georges. Historia da beleza: 0 corpo e a arte de se embelezar, do renascimento aos dias de hoje. Rio de Janeiro: Ediouro, 2006.

VILAS BOAS, Eduardo. O conceito de belo na estética grega. São Paulo: 2015.

Babieli Corsini Baccoli
Mestranda em Bioética pela Universidade do Vale do
Sapucaí (UNIVÁS).
Sapucaí (UNIVÁS).

Dênia Amélia Novato Castelli Von Atzingen Coorientadora Professora do Mestrado em Bioética UNIVÁS

Adriana Rodrigues dos Anjos Mendonça Professora Orientadora do Mestrado em Bioética UNIVÁS 